\title{
The use of computer image analysis in a Lemna minor L. bioassay
}

\author{
Robert Mazur • Krzysztof Szoszkiewicz $\mathbb{D} \cdot$ Piotr Lewicki • \\ Dawid Bedla
}

Received: 17 November 2015/Revised: 24 August 2016/Accepted: 27 August 2016/Published online: 5 September 2016

(C) The Author(s) 2016. This article is published with open access at Springerlink.com

\begin{abstract}
Our study presents a low-cost method (no expensive hardware platforms required) of quantified biomonitoring based on computer image analysis. The negative influence of toxins on surface waters was analysed. The method was verified on widespread freshwater macrophyte Lemna minor to test populations treated with non-ionic detergents. We showed that the proposed automated bioassay has a broad applicability in assessing the negative impacts of aquatic toxicants. This approach enabled fast and precise evaluation of the morphometric parameters of the duckweed test population. We observed that
\end{abstract}

Guest editors: M. T. O'Hare, F. C. Aguiar, E. S. Bakker \& K. A. Wood / Plants in aquatic systems - a 21st century perspective

\section{R. Mazur}

Department of Geoinformation Photogrammetry and Remote Sensing of the Environment, AGH University of Science and Technology in Krakow, Krakow, Poland

\section{K. Szoszkiewicz ( $₫)$}

Department of Ecology and Environmental Protection, Poznan University of Life Sciences, Poznan, Poland

e-mail: kszoszk@up.poznan.pl

\section{P. Lewicki}

Department of Tourism Policy, University of Physical Education in Krakow, Krakow, Poland

D. Bedla

Department of Ecology, Climatology and Air Protection, University of Agriculture in Krakow, Krakow, Poland growth rate of $L$. minor reacts to non-ionic detergents, which is reflected by the change in the surface area. The decrease in the growth of $L$. minor was revealed at high doses of detergents. This test proved to be highly useful, because it is well repeatable and fast in its implementation. Unlike classical bioassays, the proposed test allows the elimination of measurement errors, resulting from observers' subjectivity.

Keywords Lemna minor bioassay $\cdot$ Computer image analysis · Water pollution assessment $\cdot$ Bioindicators

\section{Introduction}

Since the 1980s, biological methods of environmental monitoring have been developed intensively (Wolna-Maruwka et al., 2012; Amini \& Kraatz, 2014; Debén et al., 2015; Fennessy et al., 2015; Harvey et al., 2015). Toxicity assessment of samples collected from the environment requires the selection of appropriate biological indicators (Kuczyńska et al., 2003; Uherek et al., 2014; Ibáñez et al., 2015), i.e. organisms particularly sensitive to the effects of specific pollutants, and the toxic effects must be indicated as clearly defined symptoms (Walker et al., 2002, 2013; Kumar et al., 2015). The majority of bioassays are based on a visual observation approach, where the observer evaluates symptoms of the toxic impact of pollution on bioindicators (Wang \& 
Freemark, 1995). A wide range of various bioindicators or biosensors (enzymes, physiological responses, etc.) have been applied to this kind of monitoring (Dobrowolski et al., 2012; Narwaria \& Saksena, 2012; Busquet et al., 2014; Wang et al., 2014). On the other hand, still a relatively limited range of biomonitoring methods are based on an automatic approach, where the subjectivity of observations is usually significantly reduced. This approach to observations often involves the use of computer image analysis methods in bioassays (Mazur \& Lewicki, 2008; Cerbin et al., 2012; Wells et al., 2012). This kind of method allows objective evaluation of the toxicity of a tested sample.

The development of image analysis procedures enables semi-automatic or fully automatic measurements. The computer records the changes and defines their nature, both qualitatively and quantitatively. In this context, it is important to find a technique for extracting information encoded in a graphical form, generally based on the characteristics of predefined features. To extract information exhibited by living organisms recorded in the form of digital images, various advanced analytical tools are used (Nowakowski et al., 2009, 2011; Sozzani et al., 2014) including advanced forms of digital simulators of brain function in the form of artificial neuron networks (Solarz et al., 2011).

The classical biotests utilising duckweed are based on dry plant biomass comparisons between the control and experimental samples. These are invasive methods and do not allow the continuation of the experiments, since the plants are harvested for biomass measurements. Another method is based on manual counting of the number of thalli ("leaves"), regardless of their size. A more reliable and more accurate method of defining the growth of biomass is comparison of the area of thalli in the control and experimental samples. This is a non-invasive method allowing the continuation of the experiment. Computer image analysis methods provide objective support for data collection (Rahaman et al., 2015; Agathokleous et al., 2016).

Our study aimed to test a low-cost approach to objective and quantified biomonitoring based on computer image analysis, reflecting the negative influence of toxins on surface waters, which can be carried out by a researcher without the need to purchase expensive hardware platforms. Our objective is to propose basic solutions for image analysis (standard digital camera) and framework software. The method was verified on test populations of the widespread freshwater macrophyte Lemna minor L., treated within a toxicity bioassay of non-ionic detergents. Our hypothesis was that the morphometric reaction of $L$. minor to non-ionic detergents could be detected with the computer image analysis approach, using a regular digital camera and Aphelion software.

\section{Materials and methods}

\section{Lemna minor axenic culture preparation}

Duckweed cultivation (stock culture) was based on Swedish standard (SIS) Lemna growth medium, prepared according to the standard procedure (OECD guideline for testing of chemicals, 2006, Lemna sp. growth inhibition test, No 221, p. 22). This protocol was followed to create an axenic culture of Lemna groups for experiment. The aquarium stock of $L$. minor axenic culture was maintained in a sterile 301 glass tank at the Laboratory of Environmental Biotechnology and Ecology, AGH University of Science and Technology in Krakow, between October 2012 and October 2013. Calibration was completed in June 2016. Each aquarium containing the sterile medium was continuously aerated. The physicochemical parameters- $\mathrm{pH}$, temperature and photoperiodwere controlled. All duckweed plants selected for the test had a high growth rate potential, were healthy, and had no visible symptoms of chlorosis on the leaves.

\section{Method calibration}

Calibration of the proposed method was carried out experimentally to test the correlations between the leaf surface area of the L. minor test populations and their weight, in an experiment consisting of 36 measuring series (with three replications). The 36 measuring series in three replications were differentiated according to the number of duckweed individuals. The image analysis procedure was carried out by measuring the frond surface area of each population, and the fresh biomass weight was estimated for them. The results were presented graphically, and the regression curve was also plotted. The correlation coefficient was 
calculated, and statistical analysis was performed for the results.

\section{Experiment with detergent}

The experiment was based on the application of nonionic detergents belonging to the Brij series to common duckweed (L. minor). Detergents are the source of many environmental problems. They deteriorate the oxygen balance in waters, and consequently disrupt self-purification processes (Mazur et al., 2013). We used Brij series detergent due to its long period of biodegradation, which has a prolonged impact on aquatic biota.

The equipment used for the toxicological tests consisted of a phytotron chamber, where beakers with the control and experimental groups were placed against a black background. The light intensity ranged from 1458 to 1499 lx; thus, it was very homogeneous on the surface (Fig. 1). The reflexes from the walls of the beakers were not eliminated; however, they did not have any influence on the changes in the growth of $L$. minor. The figure presents a digital network with the light values in 1x, measured with a Digital Lux Meter GM1020.

The images were taken using a Nikon Coolpix 995 digital camera. The camera was mounted on a stand to maintain a consistent and equal distance from the plants during each experiment. The tests were conducted in $300 \mathrm{ml}$ glass beakers, with $250 \mathrm{ml}$ of liquid in each. Ten individuals were transferred to each beaker containing different concentrations of the test detergents, and the containers were covered with Petri dishes to prevent excessive evaporation. Six concentrations for each toxicant and the control group were

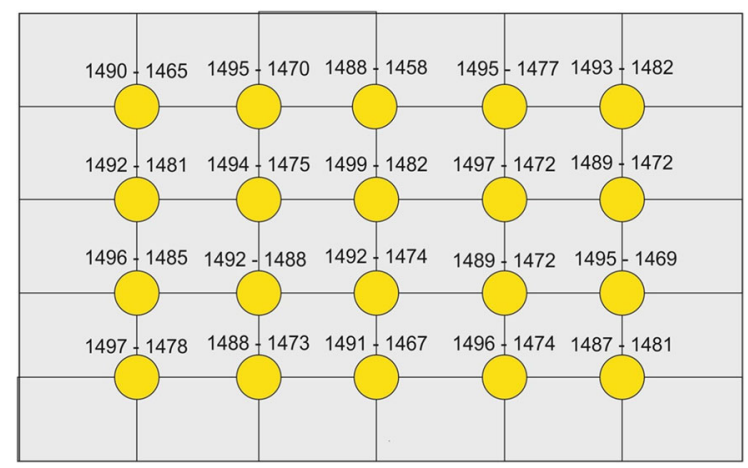

Fig. 1 The lightening conditions of the phytotron chamber with experimental groups in beakers, ranges of light intensity (in 1x) used for determination of the negative toxic effect of the bioassay. Each experiment was conducted in four replications.

Stock solutions of the toxicants were prepared in a large volume at a standardised concentration, and were diluted to lower concentrations for the chronic toxicity bioassay. The solutions of detergents Brij 58 and Brij 35 were made in geometric progression, with respective ratios of $q=1.5$ for Brij 58 and $q=1.25$ for Brij 35.

For Brij 58, the standardised concentration was $1991 \mathrm{mg} \mathrm{dm}^{-3}$, and the solutions were diluted in a geometric progression with ratio $q=1.5$ up to a concentration of $262 \mathrm{mg} \mathrm{dm}^{-3}$. Tests were performed on one control group and seven experimental groups.

For Brij 35, the standardised concentration was $800 \mathrm{mg} \mathrm{dm}^{-3}$, and this was diluted in a geometric progression with ratio $q=1.25$ up to a concentration of $210 \mathrm{mg} \mathrm{dm}^{-3}$. There were eight experimental groups and one control group. The solution coefficients were selected based on pilot tests, which enabled preliminary estimation of toxic effects for the studied detergents.

Test populations of ten individuals of $L$. minor were placed in each solution sample (one population per tray). Photographs of the L. minor plants were taken for each test group (with fixed camera settings and distances). Each image was analysed using Aphelion 3.02, and results were obtained as the average surface area of the entire test population in pixels. A macro program for automatic image analysis of a series of images was used (Fig. 2).

The algorithm begins with loading the image to be analysed. Then, a median filter was applied to reduce noise. In the next step, the colour image was converted to a monochromatic one. Breakdown of the image into RGB components was used. The blue component was used for further analysis, as it provides the highest contrast for the duckweed. In the next step, the Black Top Hat filter was used for better mining of the objects. This was followed by binarisation (corrected entropy threshold) and removal of small objects considered to be noise (erosion + image reconstruction). Next, the area with duckweed was defined (image acquisition). The tray with the duckweed was outlined in black to define parts of the plants. This enabled us to estimate the surface area for the elements inside the outline. In the final step, the objects identified by the program were outlined in 
Fig. 2 Block diagram of the duckweed detection algorithm

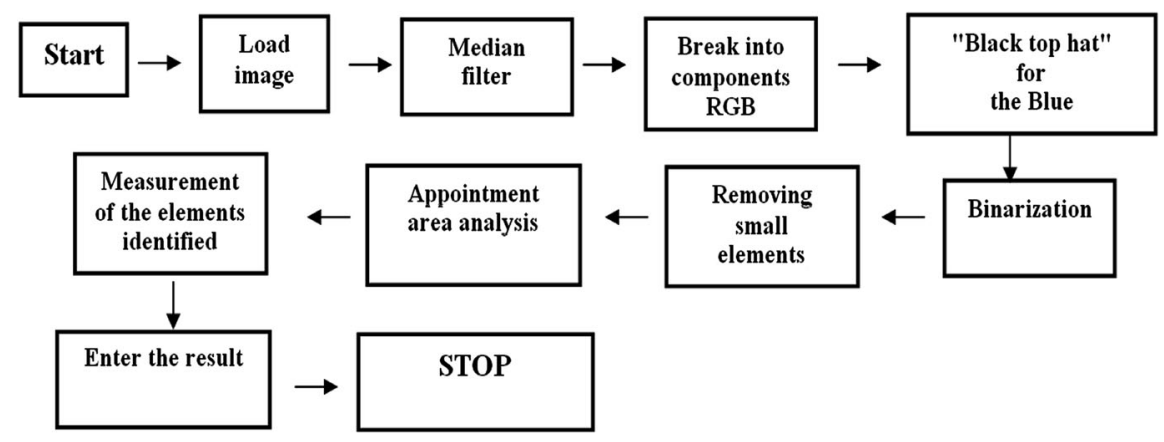

order to verify their recognition by the user, and the results were entered (Fig. 2), (Mazur \& Lewicki, 2008).

Exposure to toxicants in specific concentrations lasted 4 weeks, and five images were taken every 7 days in every test group. Glass covers were used to protect the trays with the duckweed against evaporation, while not restricting exposure to light. The experiment was carried out under strictly controlled conditions, maintaining fixed settings, while each group of L. minor was photographed: $12 / 12$ photoperiod, temperature $24 \pm 2{ }^{\circ} \mathrm{C}$.

The results were analysed and compared within groups, between groups and with the control groups, using Statistica 10 statistical software.

\section{Results}

The calibration of the method showed a strong relationship between $L$. minor surface area and the plant biomass in each group (Fig. 3). The correlation coefficient was $R^{2}=0.996$, which permits the assumption that the L. minor frond surface area is directly proportional to the mass of the test group. The

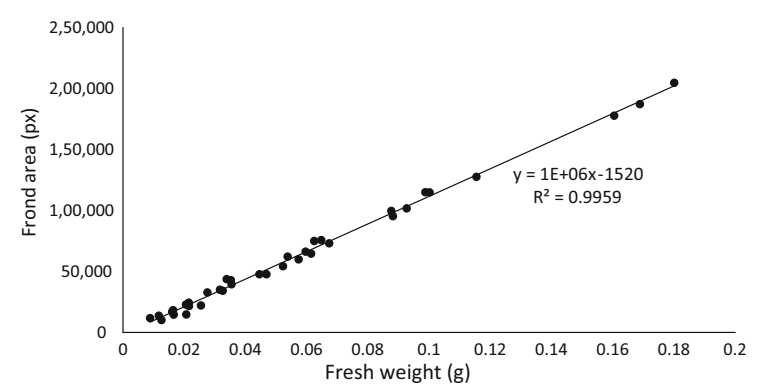

Fig. 3 Linear regression curve plot for frond area ( $\mathrm{px}$ ) and fresh weight $(\mathrm{g})$ of each experimental group of Lemna minor image analysis reflects the plant biomass very well. The results showed that, in testing the effect of pollutant toxicity on the duckweed population, both of these measures may be used interchangeably.

The principal results of the experiments on the reaction of $L$. minor to various detergent solutions (Brij 59 and Brij 38) showed that the different types of detergents resulted in different growth patterns at low doses, but high detergent applications always caused severe reduction in growth (Tables 1, 3).

The largest surface area of L. minor was recorded for the concentration of Brij 58 equal to $393 \mathrm{mg} \mathrm{dm}^{-3}$. Increasing detergent concentration beyond this point caused a significant drop in $L$. minor surface area. Plant surface areas at the highest concentration were significantly lower than in the control (Table 1; Fig. 5).

Differences in $L$. minor surface area in various stages of the experiment with Brij 58 applications are presented graphically using a box whiskers plot (Fig. 4). The non-outlier range and quartile values of L. minor are presented. This shows that a significant reaction to detergent applications requires at least a four-week period (Fig. 5).

There were significant differences in the growth dynamics of $L$. minor, depending on different detergent applications (Fig. 6).

The normal distribution of the analysed data was confirmed, and the assumption of homogeneity of variance was supported by Levene's test ( $F=1.699$, $P=0.171)$. Therefore, we used parametric ANOVA with post hoc Tukey's HSD. ANOVA showed the significance of the interaction effect, and the Tukey's test indicated a significant difference between each pair (Table 2).

Even the smallest application of Brij 35 caused a significant drop in L. minor surface area (Table 3). 
Table 1 Average value ( $\pm 1 \mathrm{SD}$ ) of Lemna minor fronds area exposed to different concentration of Brij 58 (during 1 month observation)

\begin{tabular}{|c|c|c|c|c|c|c|c|c|}
\hline \multirow{2}{*}{$\begin{array}{l}\text { Detergent concentration } \\
\left(\mathrm{mg} \mathrm{l}^{-1}\right)\end{array}$} & \multicolumn{2}{|l|}{1 week } & \multicolumn{2}{|l|}{2 weeks } & \multicolumn{2}{|l|}{3 weeks } & \multicolumn{2}{|l|}{4 weeks } \\
\hline & Mean & SD & Mean & SD & Mean & SD & Mean & SD \\
\hline Control & $17,601.3$ & 32 & $20,402.3$ & 397 & $25,788.5$ & 5223 & 33020.4 & 163 \\
\hline 262 & 18,810 & 496 & 19,073 & 818 & 25,987 & 1090 & 39453.6 & 429 \\
\hline 393 & $17,690.5$ & 1237 & $18,375.2$ & 2641 & $27,764.6$ & 1569 & 45508.9 & 1235 \\
\hline 590 & $17,570.67$ & 382 & $16,778.08$ & 606 & $24,262.33$ & 1806 & 40377 & 1345 \\
\hline 885 & $19,283.17$ & 1444 & $20,486.42$ & 2796 & $26,376.83$ & 1802 & 36608.4 & 1379 \\
\hline 1328 & $18,548.17$ & 1064 & $21,860.33$ & 2818 & $27,114.83$ & 3576 & 33515.5 & 299 \\
\hline 1991 & $18,447.42$ & 1475 & $20,519.83$ & 2444 & $24,201.92$ & 1797 & 26864.1 & 168 \\
\hline
\end{tabular}

Results of fronds area given in pixels number $(\mathrm{px})$

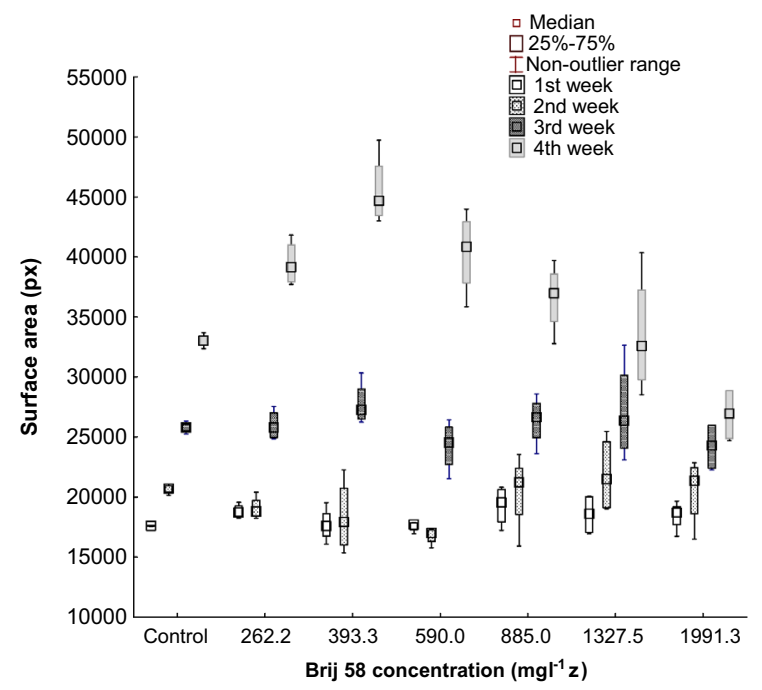

Fig. 4 Box whiskers plot: Lemna minor surface growth (in pixels) under different concentrations of the detergent during the four-week experiment

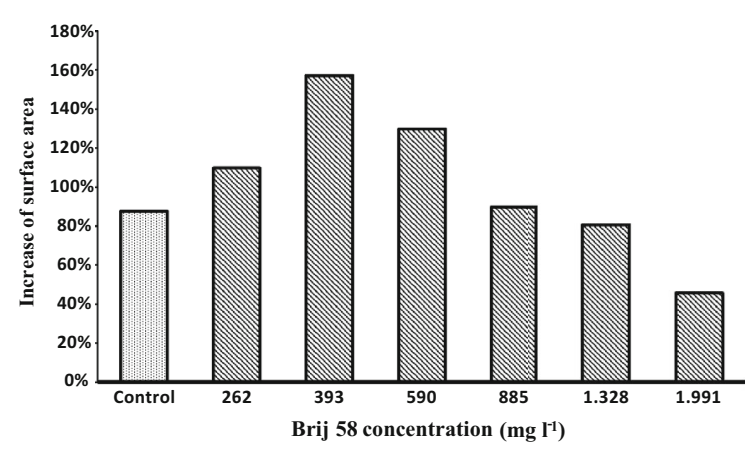

Fig. 5 The growth dynamics of Lemna minor exposed to Brij 58 solutions in each test group after 4 weeks of experiment
In analysis of the data from Brij 35 applications, the absence of normal distribution and heterogeneity of variance was detected by Levene's test $(F=1.699$, $P=0.171)$. After $\log$ transformation, the variance was still heterogeneous, so a non-parametric test (Kruskal-Wallis) was used to define the nature of the differences between the test groups and the control (Table 4).

\section{Discussion}

The method of computer image analysis was used to study acute toxicity in a modified $L$. minor bioassay. The classical method is a widely used bioassay in the assessment of surface water quality and the potential impact of toxic agents-ISO 20079:2005: 'water quality-determination of the toxic effect of water constituents and waste water on duckweed $(L$. minor)—duckweed growth inhibition test.' We showed that the L. minor bioassay supported by the computer image analysis approach for morphometric measurements of plant surface area marks new standards for a more objective and quantified assessment of the negative influence of toxins on bioindicators. Automation greatly increases the speed and accuracy of the analysis, meaning that the test can be used on an industrial scale for quality assessment of surface waters in biomonitoring.

We have confirmed that $L$. minor reacts to nonionic detergents by way of change in the plant surface area. Moreover, we found that high applications of detergents decrease the growth of L. minor, regardless 
Fig. 6 The growth dynamics of Lemna minor exposed to Brij 35 solutions in each test group after 4 weeks of experiment

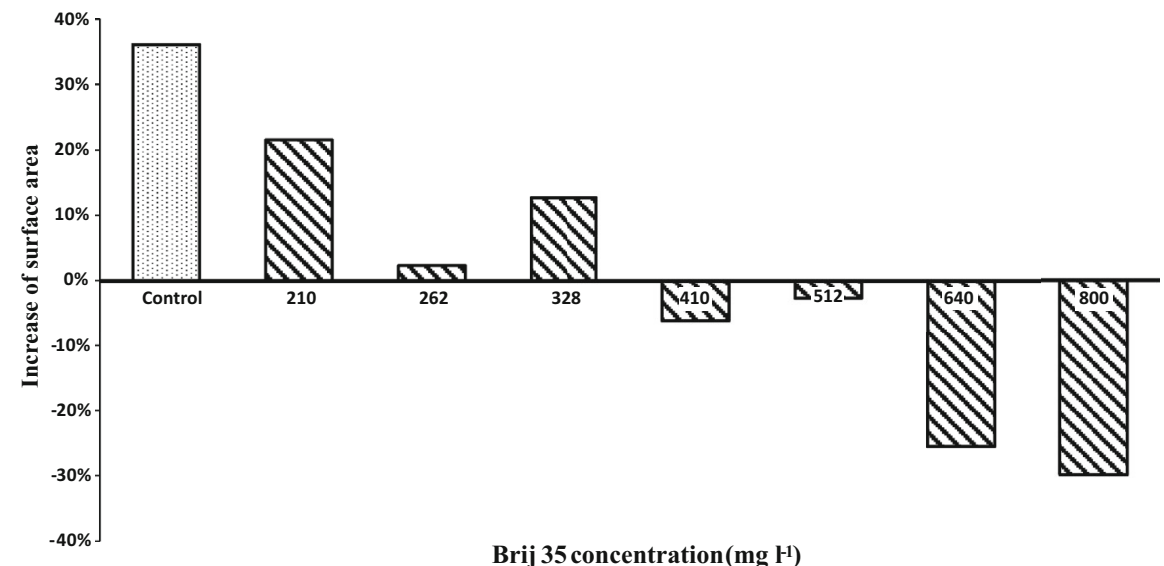

Table 2 Results of Tukey's HSD test for significance of differences between the test populations of Lemna minor exposed to different concentrations of Brij 58 solutions

$P=0.012$

\begin{tabular}{llllllll}
\hline $\begin{array}{l}\text { Detergent concentration } \\
\left(\mathrm{mg} \mathrm{l}^{-1}\right)\end{array}$ & Control & 262 & 393 & 590 & 885 & 1328 & 1991 \\
\hline Control & & 0.084 & 0.000 & 0.035 & 0.641 & 0.999 & 0.098 \\
262 & 0.084 & & 0.118 & 0.999 & 0.833 & 0.130 & 0.000 \\
393 & 0.000 & 0.118 & & 0.251 & 0.007 & 0.000 & 0.000 \\
590 & 0.035 & 0.999 & 0.251 & & 0.589 & 0.056 & 0.000 \\
885 & 0.641 & 0.833 & 0.007 & 0.589 & & 0.774 & 0.003 \\
1328 & 0.999 & 0.130 & 0.000 & 0.056 & 0.774 & & 0.068 \\
1991 & 0.098 & 0.000 & 0.000 & 0.000 & 0.003 & 0.069 & \\
\hline
\end{tabular}

Table 3 Average value ( $\pm 1 \mathrm{SD}$ ) of Lemna minor fronds area exposed to different concentration of Brij 35 (during 1 month observation)

\begin{tabular}{|c|c|c|c|c|c|c|c|c|}
\hline \multirow[t]{2}{*}{ Detergent concentration $\left(\mathrm{mg} \mathrm{l}^{-1}\right)$} & \multicolumn{2}{|l|}{1 week } & \multicolumn{2}{|c|}{2 weeks } & \multicolumn{2}{|l|}{3 weeks } & \multicolumn{2}{|c|}{4 weeks } \\
\hline & Mean & SD & Mean & SD & Mean & SD & Mean & SD \\
\hline Control & 16,977 & 167 & 19,104 & 257 & 20,991 & 111 & 23,103 & 554 \\
\hline 210 & 19,898 & 789 & 22,939 & 1096 & 24,074 & 1328 & 24,182 & 1786 \\
\hline 262 & 19,421 & 897 & 19,897 & 1441 & 20,231 & 1533 & 19,876 & 1764 \\
\hline 328 & 18,445 & 876 & 18,958 & 2443 & 20,718 & 3634 & 20,787 & 2818 \\
\hline 410 & 18,349 & 1370 & 17,073 & 1210 & 18,495 & 2655 & 17,226 & 2863 \\
\hline 512 & 19,781 & 887 & 18,269 & 552 & 19,967 & 4311 & 19,256 & 3713 \\
\hline 640 & 16,444 & 2075 & 14,263 & 635 & 14,334 & 1178 & 12,249 & 2402 \\
\hline 800 & 17,645 & 2355 & 17,642 & 2026 & 16,374 & 4363 & 12,383 & 2048 \\
\hline
\end{tabular}

Results of fronds area given in pixels number $(\mathrm{px})$

of the type of substance (both Brij 35 and Brij 58). This effect can be used in biomonitoring of water pollution.

Some differences were found between $L$. minor development patterns on treatment with different tested detergents. Brij 35 detergent had a very aggressive impact on the duckweed populations. A distinct lethal effect was found at concentrations above $640 \mathrm{mg} \mathrm{dm}^{-3}$, while concentrations of $412-509 \mathrm{mg} \mathrm{dm}^{-3}$ caused growth inhibition, and concentrations below $412 \mathrm{mg} \mathrm{dm}^{-3}$ were not toxic. Populations exposed to different concentrations of the detergent Brij 35 exhibited highly unstable growth (absence of homogeneity of variance).

Applications of Brij 58 were less toxic. Low applications of this detergent even showed a growth- 
Table 4 Results of the Kruskal-Wallis test for significance of differences between the test populations of Lemna minor exposed to different concentrations of Brij 35 solutions

\begin{tabular}{|c|c|c|c|c|c|c|c|c|}
\hline Detergent concentration $\left(\mathrm{mg} \mathrm{l}^{-1}\right)$ & Control & 209.7 & 262.1 & 327.7 & 409.6 & 512.0 & 640.0 & 800.0 \\
\hline Control & & 1.000 & 1.000 & 1.000 & 0.545 & 1.000 & 0.013 & 0.008 \\
\hline 209.7 & 1.000 & & 1.000 & 1.000 & 0.602 & 1.000 & 0.015 & 0.010 \\
\hline 262.1 & 1.000 & 1.000 & & 1.000 & 1.000 & 1.000 & 1.000 & 1.000 \\
\hline 327.7 & 1.000 & 1.000 & 1.000 & & 1.000 & 1.000 & 0.807 & 0.602 \\
\hline 409.6 & 0.545 & 0.602 & 1.000 & 1.000 & & 1.000 & 1.000 & 1.000 \\
\hline 512.0 & 1.000 & 1.000 & 1.000 & 1.000 & 1.000 & & 1.000 & 1.000 \\
\hline 640.0 & 0.013 & 0.015 & 1.000 & 0.807 & 1.000 & 1.000 & & 1.000 \\
\hline 800.0 & 0.008 & 0.010 & 1.000 & 0.602 & 1.000 & 1.000 & 1.000 & \\
\hline
\end{tabular}

$P=0.038$

stimulating effect. Concentrations of 393 and $590 \mathrm{mg} \mathrm{dm}^{-3}$ of Brij 58 produced statistically significant differences in biomass growth compared with the control. This was a stimulatory effect, which is confirmed by literature reports on the impact of detergents on eutrophication as a result of introducing biogenic elements to surface waters (Mazur et al., 2013). Applications of Brij 58 at a concentration of $1991 \mathrm{mg} \mathrm{dm}^{-3}$ reduced the growth rate of L. minor, exhibiting a toxic effect.

In our study, we have focused on L. minor, which is a widespread freshwater macrophyte that is advantageous for biomonitoring (Wang \& Freemark, 1995). Brij applications have been widely used in toxicological experiments. Most toxicity tests for Brij detergents have been performed on higher organisms, but safety data sheets for chemical substances also require specification of acute toxicity for aquatic organisms (Noudeh et al., 2011; Williams et al., 2011; BeneitoCambra et al., 2013; Li et al., 2013).

Our study showed the applicability of computer image analysis methods for toxicity bioassays with $L$. minor based on measuring the change in plant surface area. Moreover, the macro program analyses a series of images from a particular catalogue, and the data obtained are returned in a Microsoft Excel spreadsheet. This provides an extensive and comprehensive database that can be used in further statistical analyses of the investigated objects.

We have found that computer image analysis may provide important support in bioassays. Although the use of computer image analysis in water monitoring is currently gaining recognition, still few automated systems have been created and brought into use in biomonitoring (Streb et al., 2002; Akagi et al., 2014). The automated $L$. minor bioassay provides a new tool for assessment of the negative influence of aquatic toxicants. Present trends in aquatic ecotoxicology require toxicological assessment to be carried out with a wide range of bioassays (Garric et al., 1993; Zgorska et al., 2011; Forni et al., 2012; Testolin et al., 2012).

\section{Conclusions}

1. The growth rate of $L$. minor is affected by nonionic detergents, as reflected by changes in the surface area.

2. A high concentration of detergents always reduced the growth of L. minor.

3. The morphometric reaction of L. minor to nonionic detergents can be detected with the computer image analysis approach, using framework software and basic equipment.

4. The new L. minor bioassay with the application of computer image analysis for morphometric measurements of the plant surface area marks new standards for more objective and quantified assessment of the negative influence of toxins on bioindicators. The test can be used on an industrial scale for quality assessment of surface waters in biomonitoring.

Open Access This article is distributed under the terms of the Creative Commons Attribution 4.0 International License (http:// creativecommons.org/licenses/by/4.0/), which permits unrestricted use, distribution, and reproduction in any medium, provided you give appropriate credit to the original 
author(s) and the source, provide a link to the Creative Commons license, and indicate if changes were made.

\section{References}

Agathokleous, E., A. C. Mouzaki-Paxinou, C. J. Saitanis, E. Paoletti \& W. J. Manning, 2016. The first toxicological study of the antiozonant and research tool ethylene diurea (EDU) using a Lemna minor L. bioassay: hints to its mode of action. Environmental Pollution 213: 996-1006.

Akagi, J., F. Zhu, C. J. Hall, K. E. Crosier, P. S. Crosier \& D. Wlodkowic, 2014. Integrated chip-based physiometer for automated fish embryo toxicity biotests in pharmaceutical screening and ecotoxicology. Cytometry Part A 85: 537-547.

Amini, K. \& H. B. Kraatz, 2014. Recent advances and developments in monitoring biological agents in water samples. Reviews in Environmental Science and Biotechnology 14: 23-48.

Beneito-Cambra, M., J. M. Herrero-Martínez \& G. RamisRamos, 2013. Analytical methods for the characterization and determination of nonionic surfactants in cosmetics and environmental matrices. Analytical Methods 5: 341-354.

Busquet, F., R. Strecker, J. M. Rawlings, S. E. Belanger, T. Braunbeck, G. J. Carr \& M. Halder, 2014. OECD validation study to assess intra and inter-laboratory reproducibility of the zebrafish embryo toxicity test for acute aquatic toxicity testing. Regulatory Toxicology and Pharmacology 69: 496-511.

Cerbin, S., J. Dach, K. Nowakowski, K. Pilarski, P. Boniecki, J. Przybyl \& A. Lewicki, 2012. Possibilities of neural image analysis implementation in monitoring of microalgae production as a substrate for biogas plant. 4th international conference on digital image processing (ICDIP 2012) book series: proceedings of SPIE. 8334: 83342A.

Debén, S., J. R. Aboal, A. Carballeira, M. Cesa, C. Real \& J. A. Fernández, 2015. Inland water quality monitoring with native bryophytes: a methodological review. Ecological Indicators 53: 115-124.

Dobrowolski, J. W., M. Śliwka \& R. Mazur, 2012. Laser biotechnology for more efficient bioremediation, protection of aquatic ecosystems and reclamation of contaminated areas. Journal of Chemical Technology and Biotechnology 87: 1354-1359.

Fennessy, S., C. Ibañez, A. Munné, N. Caiola, N. Kirchner \& C. Sola, 2015. Biological Indices Based on Macrophytes: An Overview of Methods Used in Catalonia and the USA to Determine the Status of Rivers and Wetlands. Springer, Cham.

Forni, C., R. Braglia, F. J. M. Harren \& S. M. Cristescu, 2012. Stress responses of duckweed (Lemna minor L.) and water velvet (Azolla filiculoides Lam.) to anionic surfactant sodium-dodecyl-sulphate (SDS). Aquatic Toxicology 110: 107-113.

Garric, J., E. Vindimian \& J. F. Ferard, 1993. Ecotoxicology and wastewater: some practical applications. Science of the Total Environment 134: 1085-1103.

Harvey, E., T. S. Kratzer \& P. Philipson, 2015. Satellite-based water quality monitoring for improved spatial and temporal retrieval of chlorophyll-a in coastal waters. Remote Sensing of Environment 158: 417-430.

Ibáñez, C., N. Caiola, R. Trobajo, A. Nebra \& L. Rovira, 2015. Biological indicators to assess the ecological status of river-dominated estuaries: the case of benthic indicators in the Ebro river estuary. In Ginebreda, A. \& N. Prat (eds), Experiences from Surface Water Quality Monitoring. Springer, Berlin.

Kuczyńska, A., L. Wolska \& J. Namieśnik, 2003. Application of bioassays in environmental research, new horizons in analytics and environmental monitoring, CDAiMŚ (CEEAM).

Kumar, A., M. B. M. Colton, M. Springer \& F. A. Trama, 2013. Macroinvertebrate communities as bioindicators of water quality in conventional and organic irrigated rice fields in Guanacaste, Costa Rica. Ecological Indicators 29: 68-78.

Li, X., H. Li, C. Y. Chen, J. T. Li \& F. Liu, 2013. Acute toxicity of different type pesticide surfactants to Daphnia magna. The Journal of Applied Ecology 24: 2319-2324.

Mazur, R. \& P. Lewicki, 2008. Application of the computer image analyses in the embryotests as a new method of water biomonitoring. Polish Journal of Environmental Studies 17: 409-412.

Mazur, R., A. Wagner \& M. Zhou, 2013. The application of the Lymnaea stagnalis embryo-test in the toxicity bioindication of surfactants in fresh waters. Ecological Indicators 30: 190-195.

Narwaria, Y. S. \& D. N. Saksena, 2012. Acute toxicity bioassay and behavioural responses induced by sodium fluoride in freshwater fish Puntius sophore (bloch). Fluoride 45: 7-12.

Noudeh, G. H., P. Khazaeli, E. Behravan, M. A. Afzadi, A. Dehghan-Noudeh \& M. Hassani, 2011. Evaluating the toxicity of permeability enhancers of polyethylene glycol Brij ethers surfactants group on cellular membranes and some of their physicochemical properties. African Journal of Biotechnology 10: 9931-9938.

Nowakowski, K., P. Boniecki \& J. Dach, 2009. The identification of mechanical damages of Kernels basis on neural image analysis. International conference on digital image processing, Bangkok, MAR 07-09, proceedings: 412-415.

Nowakowski, K., P. Boniecki, R. J. Tomczak \& B. Raba, 2011. Identification process of corn and barley kernels damages using neural image analysis. 3rd international conference on digital image processing Chengdu, APR 15-17, proceedings of SPIE: $80090 \mathrm{C}$.

Rahaman, M. M., D. Chen, Z. Gillani, C. Klukas \& M. Chen, 2015. Advanced phenotyping and phenotype data analysis for the study of plant growth and development. Frontiers in Plant Science 6: 619.

Solarz, P., M. Stanisz, P. Boniecki, A. Przybylak, D. Lisiak \& A. Ludwiczak, 2011. Artificial neural network analysis of ultrasound image for the estimation of intramuscular fat content in lamb muscle. African Journal of Biotechnology 10: $11792-11796$.

Sozzani, R., W. Busch, E. P. Spalding \& P. N. Benfey, 2014. Advanced imaging techniques for the study of plant growth and development. Trends in Plant Science 19(5): 304-310.

Streb, C., P. Richter, M. Ntefidou, M. Lebert \& D. P. Hader, 2002. ECOTOX-biomonitoring based on real time movement analysis of unicellular organisms. Journal of Gravitational Physiology 9: 345-346. 
Testolin, R. C., V. Tischer, A. O. S. Lima, S. Cotelle, J. F. Férard \& C. M. Radetski, 2012. Aquatic ecotoxicology assessment of a new natural formicide. Environmental Science and Pollution Research 19: 2186-2194.

Uherek, C. B. \& F. B. Pinto Gouveia, 2014. Biological monitoring using macroinvertebrates as bioindicators of water quality of Maroaga stream in the Maroaga Cave System, Presidente Figueiredo, Amazon, Brazil. International Journal of Ecology 2014: 1-7.

Walker, C. H., S. P. Hopkin, R. M. Sibly \& D. B. Peakall, 2002. Principles of Ecotoxicology. CRC Press, Taylor and Francis Group, New York: 175-194.

Walker, T. R., D. Macaskill \& P. Weaver, 2013. Blue mussels (Mytilus edulis) as bioindicators of stable water quality in Sydney Harbour during remediation of the Sydney Tar Ponds, Nova Scotia, Canada. Ecological Indicators 48: 258-371.

Wang, W. C. \& K. Freemark, 1995. The use of plants for environmental monitoring and assessment. Ecotoxicology and Environmental Safety 30: 289-301.

Wang, Y., T. Suzek, J. Zhang, J. Wang, S. He, T. Cheng \& S. H. Bryant, 2014. PubChem bioAssay: 2014 update. Nucleic Acids Research 42: D1075-D1082.
Wells, D. M., A. P. French, A. Naeem, O. Ishaq, R. Traini, H. Hijazi \& T. P. Pridmore, 2012. Recovering the dynamics of root growth and development using novel image acquisition and analysis methods. Philosophical Transactions of the Royal Society B 367: 1517-1524.

Williams, E. S., J. P. Berninger \& B. W. Brooks, 2011. Application of chemical toxicity distributions to ecotoxicology data requirements under REACH. Environmental Toxicology and Chemistry 30: 1943-1954.

Wolna-Maruwka, A., A. Schroeter-Zakrzewska \& J. Dach, 2012. Analysis of the growth and metabolic activity of microorganisms in substrates prepared on the base of sewage sludge and their impact on growth and flowering of garden Verbena. Fresenius Environmental Bulletin 21: 325-336.

Zgorska, A., A. Arendarczyk \& E. Grabińska-Sota, 2011. Toxicity assessment of hospital wastewater by the use of a biotest battery. Archives of Environmental Protection 37: 55-61. 